\title{
Heartbeat: Renin-angiotensin system blockade for prevention of cardiovascular disease
}

Angiotensin converting enzyme inhibitors (ACEI) and angiotensin receptor blocker (ARB) medications both target the reninangiotensin system (RAS) and both are recommended in treatment of several types of cardiovascular disease (CVD). However, the optimal choice between these 2 groups of medications for prevention of CVD is not well established. Potier and colleagues ${ }^{1}$ compared the relative effectiveness of ACEIs versus ARBs for prevention of adverse outcomes in over 40 thousand patients at high cardiovascular risk enrolled in the observational Reduction of Atherothrombosis for Continued Health (REACH) registry. This international registry includes adults over age 45 years with known CVD or with at least 3 risk factors for atherosclerosis; the current analysis includes only those subjects taking an ACEI or ARB at baseline who had 4-year follow-up data. Medical therapy was provided by individual physician practices for standard indications. Baseline conditions included a history of hypertension (91\%), heart failure (16\%), atrial fibrillation/flutter (11\%), coronary artery disease (60\%), cerebrovascular disease (26\%) and diabetes (49\%). Overall, the propensity score adjusted (and matched) results suggest that ARB use is associated with a $10 \%$ lower rate of CVD events compared to ACEI use (figure 1). However, this difference was only seen in those with established atherosclerosis, not those with risk factors alone.

In the accompanying editorial, Furukawa $^{2}$ notes that: 'There remain two important controversies regarding the effects of RAS inhibitors; one question is whether RAS inhibitors have anti-atherosclerotic effects beyond lowering blood pressure' The other important question is whether ACEIs and ARBs have equivalent effectiveness in preventing cardiovascular events.' His figure nicely summarises differences between and potential mechanisms of benefit of RAS blockade (figure 2) He concludes: 'The current report by the $\mathrm{REACH}$ investigators reminds us of the

Correspondence to Professor Catherine M Otto, Division of Cardiology, University of Washington, Seattle, Washington 98195, USA; cmotto@uw.edu, cmotto@u.washington.edu

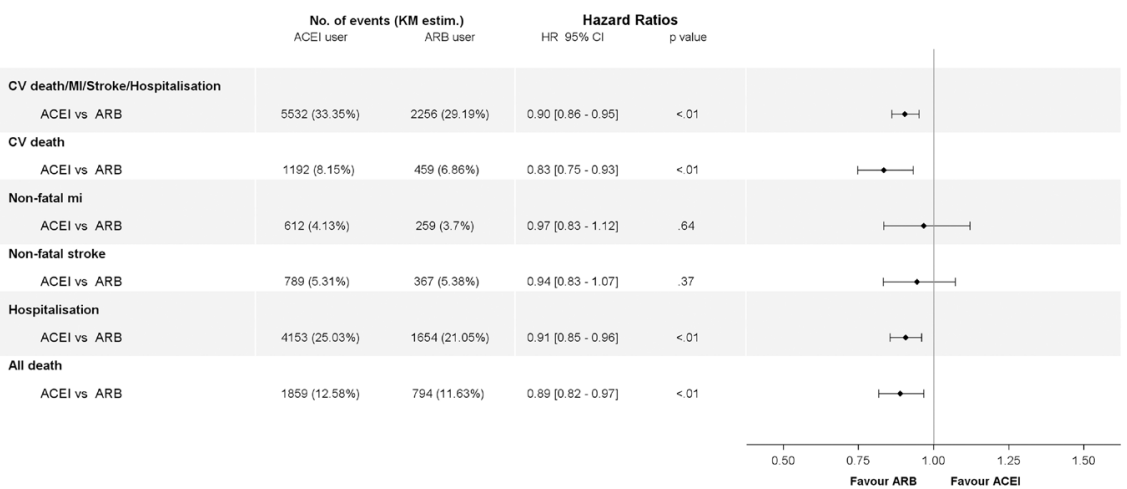

Figure 1 Risks of outcomes in ACEls versus ARBs cohorts, in propensity score-adjusted analysis. ACEls, ACE inhibitors; ARB, angiotensin receptor blockers; CV, cardiovascular; MI, myocardial infarction. ${ }^{1}$

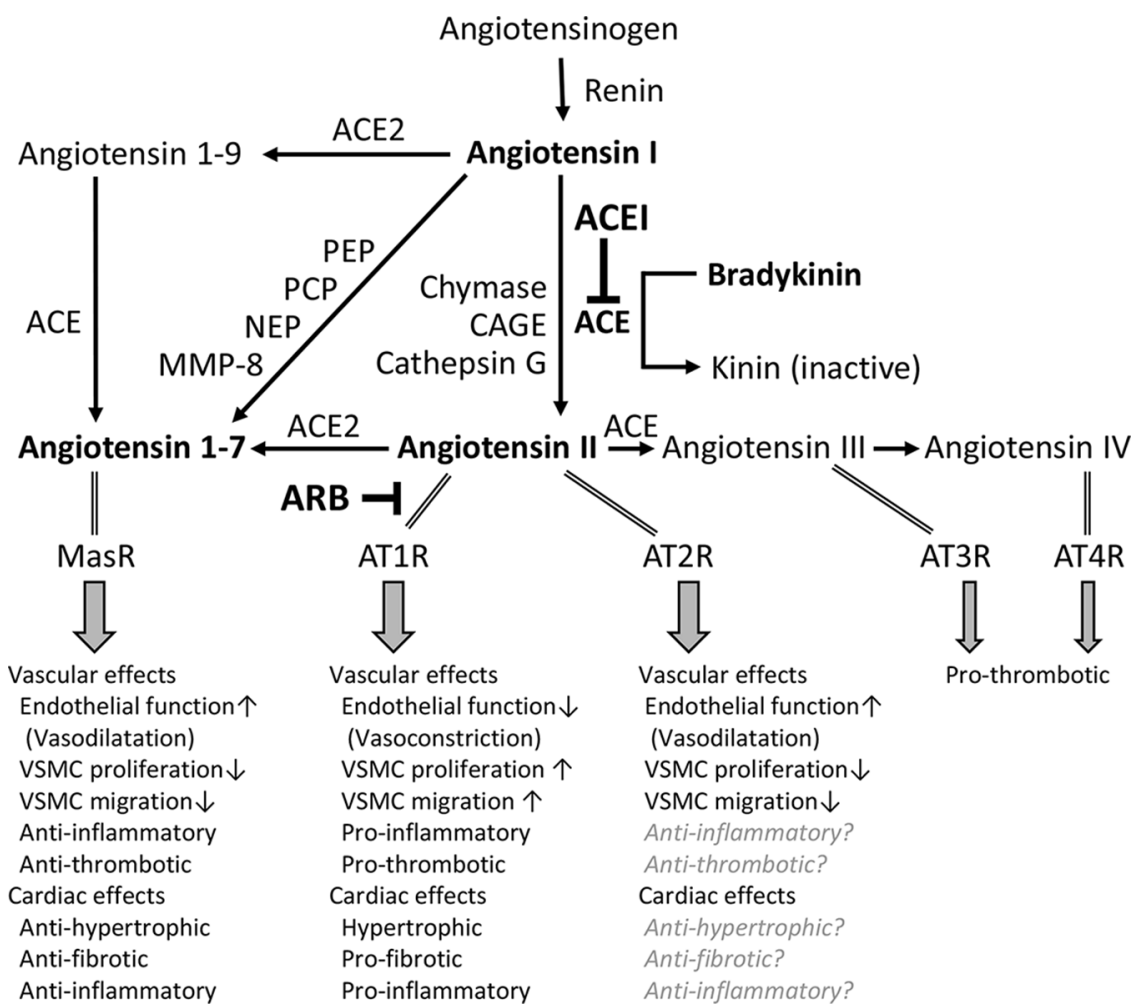

Figure 2 Renin-angiotensin system (RAS) and related molecules. Many molecules influence the effects of RAS activation. Angiotensin II and AT1R are key molecules of the major deleterious pathway in the cardiovascular system. Counteracting pathways with a cardiovascular protective property also exist. There are controversies about the effects of AT2R signalling which are indicated by grey italic characters. ARB, angiotensin receptor blocker; AT1R, angiotensin type one receptor; AT2R, angiotensin type two receptor; AT3R, angiotensin type three receptor; AT4R, angiotensin type four receptor; CAGE, chymostatin-sensitive angiotensinII-generating enzyme; MasR, Mas receptor; MMP-8, matrix metalloproteinase-8; NEP, neutral endopeptidase; PCP, prolyl carboxypeptidase; PEP, prolyl endopeptidase; VSMC, vascular smooth muscle cell. 


\section{Initiator of anticoagulant treament:}

Sets indication for anticoagulation;

Chooses anticoagulant, based also on patient preferences;

Decides on need of proton pump inhibitor;

Baseline hemoglobin, renal and liver function;

Provides education;

Hands out anticoagulation card;

Organises follow-up (when, by whom, what?);

Remains responsible coordinator for follow-up.

\section{first FU: 1 month}

\section{Follow-up: GP; anticoagulant clinic; initiator of therapy;}

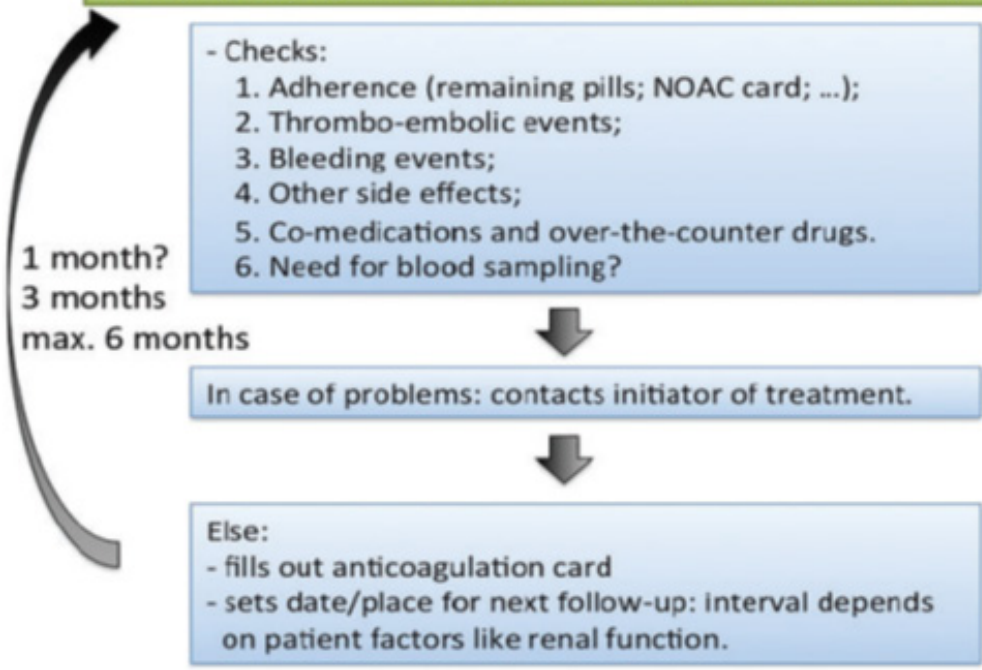

Figure 3 Suggested initiation and structured follow-up pathway of patients on NOACs. Reproduced with permission from Heidbuchel et al (Europace 2015; 17: 1467-507) and Oxford University Press. need to re-evaluate the relative effectiveness of ARBs versus ACEIs in various settings of cardiovascular prevention.'

A challenge in healthcare delivery is ensuring patients initiate and continue to take prescribed medications. This has been a particular concern in the transition from monitored vitamin $\mathrm{K}$ antagonist (VKA) anticoagulation to unmonitored therapy with a novel oral anticoagulant (NOAC). In a retrospective cohort study from Canada, failure to continue NOAC therapy (non-persistence) at 6 months was observed in $36.5 \%$ of over 15 thousand patients prescribed dabigatran and $31.9 \%$ of over 10 thousand patients prescribed rivaroxaban. ${ }^{3}$ Not surprisingly the risk of cerebrovascular events was higher in those with non-persistence compared with those who continued on NOAC therapy. Of course, these observations need to be considered in the context that suboptimal adherence to VKA therapy, as evidenced by a low time in therapeutic range, also is typical.
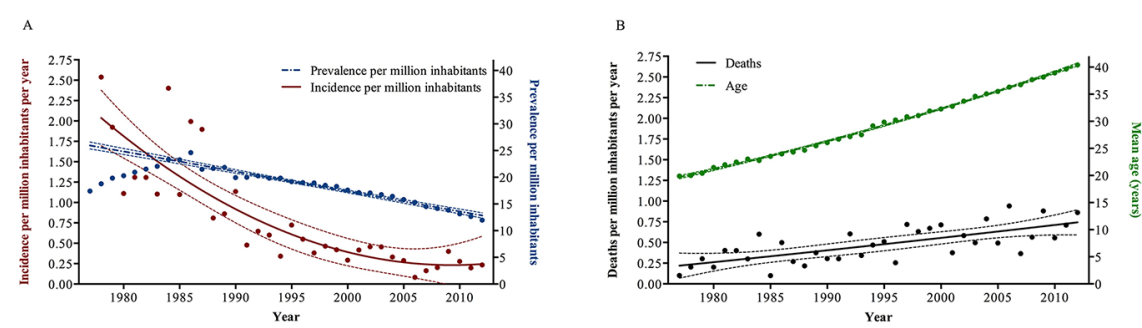

Figure 4 Epidemiological changes in Eisenmenger syndrome (ES) in the Nordic countries from 1977 to 2012. (A) Incidence and prevalence and (B) mean age of the ES population and the rate of death. $^{5}$
Although the reasons for non-persistence could not be determined in this Canadian study, Hendrick, Gallagher and Sanders ${ }^{4}$ advocate that: 'A comprehensive integrated approach is needed to incorporate stroke prevention into daily practice, including structured follow-up in those patients on NOAC treatment to evaluate and prevent non-persistence and adverse effects of therapy' (figure 3). 'Although patients on NOAC treatment may not require routine blood monitoring to the same degree as those on VKA antagonists, it is clear that this should not be translated to a lack of requirement for monitoring of the individual.'

Diagnosis and management of congenital heart disease (CHD) has dramatically improved over the past few decades. A retrospective study of 714 patients with Eisenmenger syndrome (ES) in the Nordic region, showed a decrease in incidence of ES from 2.5/million inhabitants/ year in 1977 to $0.2 /$ million inhabitants/ year in 2012. In addition, median age at death increased from 27.7 years between 1977 and 992 to 46.3 years from July 2006 to $2012^{5}$ (figure 4 ).

In an editorial, Kempny, Dimopoulus and Gatzoulis ${ }^{6}$ remind us that in patients established ES surgical intervention is prohibitively risky with no evidence for clinical benefit. Thus, the declining prevalence of ES reflects improved early diagnosis and treatment in infancy, preventing the development of ES in the first place. In contrast, longer survival times in those with established ES likely are related to conservative supportive therapies in conjunction with targeted therapy for pulmonary arterial hypertension (PAH). They conclude: 'We are likely to encounter a change in demographics within the PAH-CHD population in the years to come, with more patients presenting with $\mathrm{PAH}$ after defect repair, a condition associated with increased mortality in patients

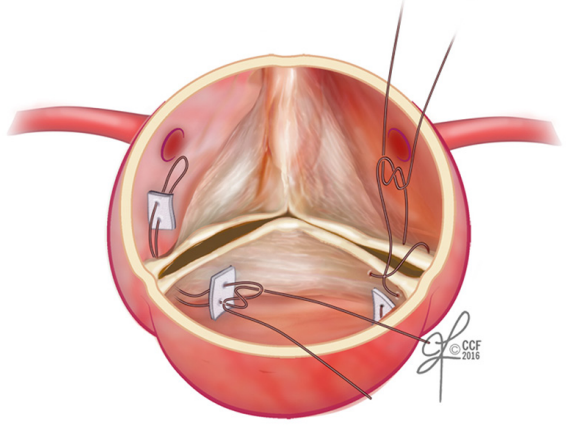

Figure 5 Schematic illustration of a standard aortic valve repair technique. ${ }^{7}$ 


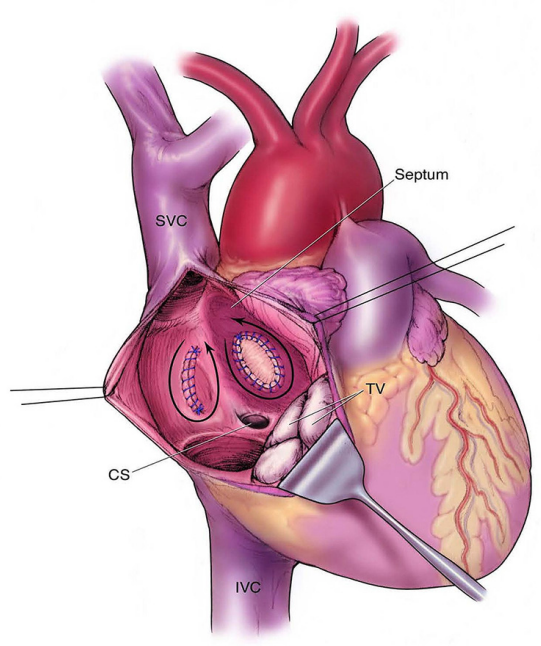

Figure 6 Illustrates two of the potential circuits for intra-atrial reentrant tachycardia (IART). The scars/patches act as discrete boundaries, providing a fundamental component for the initiation and maintenance of reentrant atrial arrhythmia. Similar to typical atrial flutter in the normal heart, the most common atrial arrhythmia in this group also relies on the isthmus of atrial tissue between the sub-pulmonic atrioventricular valve and the inferior vena cava.

with CHD. Efforts should, therefore, be directed into better understanding and improving the management of
non-Eisenmenger PAH-CHD, as well as that of special cohorts such as patients with Down syndrome, while supporting low/middle-income countries to reduce the incidence of ES.'

A congenital bicuspid aortic valve is present in about $1 \%$ of the population and nearly all of these patients will require aortic valve intervention at some point in their lifetime. In this issue of Heart, Masri and colleagues ${ }^{7}$ from the Cleveland Clinic present a systemic review of the natural history and clinical outcomes of patients with bicuspid aortic valve disease. This comprehensive literature summary provides clinicians a quick overview of this condition along with an introduction to newer surgical approaches, and lays the framework for future research on this common condition (figure 5).

The Education in Heart article in this issue $^{8}$ focuses on diagnosis and management of acute arrhythmias in the adults with CHD. This topic is relevant for clinicians because these patients often initially present emergently to the primary care provider or general cardiologist. Mechanisms of arrhythmias in this patient group are illustrated nicely as well (figure 6).

Competing interests None declared.

Provenance and peer review Commissioned; internally peer reviewed. (c) Article author(s) (or their employer(s) unless otherwise stated in the text of the article) 2017. All rights reserved. No commercial use is permitted unless otherwise expressly granted.

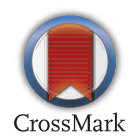

To cite Otto CM. Heart 2017;103:1305-1307.

Heart 2017;103:1305-1307.

doi:10.1136/heartjnl-2017-312121

\section{REFERENCES}

1 Potier L, Roussel R, Elbez Y, et al. Angiotensin-converting enzyme inhibitors and angiotensin receptor blockers in high vascular risk. Heart 2017;103:1339-46.

2 Furukawa Y. Angiotensin-converting enzyme inhibitors versus receptor blockers: is one better than the other for cardiovascular prevention? Heart 2017;103:1310-2.

3 Jackevicius CA, Tsadok MA, Essebag V, et al. Early nonpersistence with dabigatran and rivaroxaban in patients with atrial fibrillation. Heart 2017;103:1331-8.

4 Hendriks JM, Gallagher C, Sanders P. Ensuring adherence to therapy with anticoagulation in patients with atrial fibrillation. Heart 2017;103:1308-9.

5 Hjortshøj CS, Jensen AS, Sørensen K, et al. Epidemiological changes in Eisenmenger syndrome in the Nordic region in 1977-2012. Heart 2017:103:1353-8.

6 Kempny A, Dimopoulos K, Gatzoulis MA. Declining incidence and prevalence of Eisenmenger syndrome in the developed world: a triumph of modern medicine. Heart 2017;103:1313-4.

7 Masri A, Svensson LG, Griffin BP, et al. Contemporary natural history of bicuspid aortic valve disease: a systematic review. Heart 2017;103:1323-30.

8 McLeod CJ. Acute arrhythmias in adults with congenital heart disease. Heart 2017;103:1380-7. 